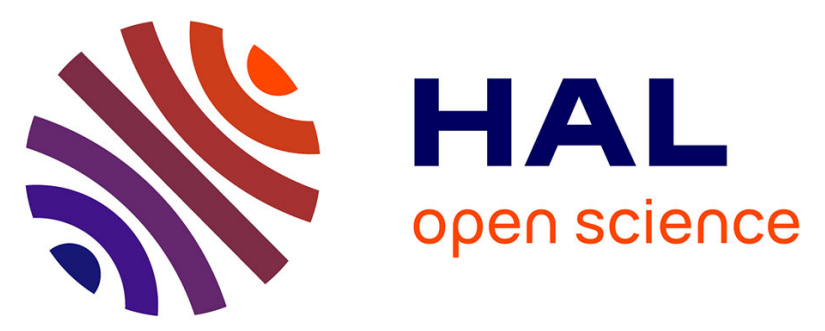

\title{
IMPORTANCE OF MOMENTUM DEPENDENT INTERACTIONS FOR THE EXTRACTION OF THE NUCLEAR EQUATION OF STATE FROM HIGH ENERGY HEAVY ION COLLISIONS
}

\author{
Joerg Aichelin, A. Rosenhauer, G. Peilert, H. Stoecker, W. Greiner
}

\section{To cite this version:}

Joerg Aichelin, A. Rosenhauer, G. Peilert, H. Stoecker, W. Greiner. IMPORTANCE OF MOMENTUM DEPENDENT INTERACTIONS FOR THE EXTRACTION OF THE NUCLEAR EQUATION OF STATE FROM HIGH ENERGY HEAVY ION COLLISIONS. Journal de Physique Colloques, 1987, 48 (C2), pp.C2-165-C2-174. 10.1051/jphyscol:1987224 . jpa-00226490

HAL Id: jpa-00226490 https://hal.science/jpa-00226490

Submitted on 1 Jan 1987

HAL is a multi-disciplinary open access archive for the deposit and dissemination of scientific research documents, whether they are published or not. The documents may come from teaching and research institutions in France or abroad, or from public or private research centers.
L'archive ouverte pluridisciplinaire HAL, est destinée au dépôt et à la diffusion de documents scientifiques de niveau recherche, publiés ou non, émanant des établissements d'enseignement et de recherche français ou étrangers, des laboratoires publics ou privés. 
IMPORTANCE OF MOMENTUM DEPENDENT INTERACTIONS FOR THE EXTRACTION OF THE NUCLEAR EQUATION OF STATE FROM HIGH ENERGY HEAVY ION COLLISIONS

J. AiCHELIN, A. ROSENHAUeR* ${ }^{*}$ G. PEILERT ${ }^{*}, \mathrm{H}$. STOECKER $^{*}$ and W. GREINER*

Institut für Theoretische Physik der Universität Heidelberg and Max-Planck-Institut für Kernphysik, D-6900 Heidelberg, F.R.G.

"Institut für Theoretische Physik, Johann wolfgang Goethe Universität Frankfurt, D-6000 Frankfurt, F.R.G.

Abstract: We demonstrate that momentum dependent nuclear interactions (MDI) have a large effect on the dynamics and on the observables of high energy heavy ton collistons: Pion and kaon yields are strongly suppressed by MDI. This effect is much stronger than the supression due to a hard local potential, which introduces an ambiguity into the recent extraction of the nuclear equation of state from pion (and kaon) yields. Also the transverse momentum distributions exhibit a strong dependence on the MDI. The collective flow angles and the deuteron to proton ratios, on the other hand, are rather insensitive to the MDI. They may therefore be related directly to the nuclear equation of state at high density. Simultaneous measurements of these observables in heavy ion collisions can give clues on the nuclear equation of state at densities of interest for supernova collapse and neutron star stabllity.

The current studies of high energy heavy fon collisions are of broad scientific interest. This is for two reasons: first, they offer a unique testing ground for newly developped methods to study the behaviour of strongly interacting quantum systems with finite particle number far from the groundstate. Secondly one hopes that information on the equation of state of dense nuclear matter can be extracted from the experimental data. This knowledge is essential for an understanding of the collapse of supernovae, for neutron star stability and for the 
onset of a possible transition from hadron matter to the quark gluon plasma.

It has been proposed for many years to use pion production and the collective flow in central high energy collisions to investigate the nuclear EOS at high denst ty and temperature ${ }^{1-4}$. The microscopic Vlasov Uehling Uhlenbeck (VUU) theory ${ }^{3-5}$ has exhiblted the sensitivity of the pion ylelds ${ }^{1,3}$, subthreshold kaon yields ${ }^{5}$ and the collective flow $1,3,4$ to the nuclear EOS. It has recently been suggested ${ }^{6}$ to test whether this sensitivity to the equation of state might be distorted by introducing momentum dependent interactions (MDI) into the theory. Such interactions had been Implemented into the classical "molecular dynamics" 7 and the time dependent meson field approach ${ }^{8}$ which predict $^{7-9}$ a collective sidewards flow in qualitative agreement with the data 10

Here we demonstrate the importance of the momentum dependent interactions using an extension of the VU approach, dubbed "quantum molecular dynamics" (OMD) ${ }^{11}$, which combines the important quantum features of the VUU theory, namely the Paull principle, stochastic scattering and particle production ${ }^{1,3-5}$, with the long range $N$ - body correlations found in the classical molecular dynamics method ${ }^{7-9}: N$ gaussian wavepackets with a finlte width in configuration and momentum space move on trajectories given by the $\mathbf{N - b o d y}$ interaction, following Hamilton's equations. $d p / d t=-d H / d q . d q / d t=p / m+d U / d p$. Two wave packets can also scatter stochastically at short distances. The width of the wave packets allows for a computation of the phase space occupancy (1-f) which determines the Paull blocking probability 11 of attempted collisions in the same way as in the VUU approach ${ }^{3-5}$. Deltas, plons and kaons are produced using the elementary scattering cross sections in the same way as in the VU approach ${ }^{3-5,11}$.

The potential $U$ is given by

$$
u=\sum_{1} t_{1} \delta\left(r_{1}-r_{i}^{j}\right)+t_{2}\left(\sum_{1}^{\Sigma} \delta\left(r_{1}-r_{i}\right)\right)^{\alpha}+u_{c o u l}+U(\Delta p) \delta\left(r_{1}-r_{i}^{j}\right) \text {. }
$$


The momentum dependent term $U(\Delta p)$ is taken ${ }^{12}$ from the measured energy dependence of the proton - nucleus optical potential in the range of $10 \mathrm{MeV}<E_{\mathrm{Kin}}<1 \mathrm{GeV}$. (It can be parametrized as $U(\Delta p)=1.57$ $\left(\ln \left[1+5 \times 10^{-4} \Delta p^{2}\right]\right)^{2} p / p_{0}[M e V]$, where the relative momentum $\Delta p=p_{1}-p_{2}$ is given in units of MeV/C. Nuclear matter calculations ${ }^{12}$ guide the assumed density dependence of $U(\Delta p)$.) The dU/dp term in the equations of motion yields an effective nucleon mass of $\mathrm{m}^{*} / \mathrm{m}=0.75$ at the Fermi momentum, and $\mathrm{m}^{*} / \mathrm{m}=0.95-0.98$ at relative momenta in the BEVALAC energy region. $E_{\text {lab }}=0.8 \mathrm{GeV} / \mathrm{n}$. The mean potential fleld can be cast into a form which explicitly shows the density dependence of the interaction in infinite nuclear matter at $T=0$;

$$
U(\rho)=\alpha \rho / \rho_{0}+\beta\left(\rho / \rho_{0}\right)^{\gamma}+\delta\left(\ln ^{2}\left(1+\epsilon\left(\rho / \rho_{0}\right)^{2 / 3}\right) \rho / \rho_{0}\right.
$$

This can be used to compute the corresponding dens1ty dependence of the compressional energy per nucleon (the 'cold EOS') ${ }^{1-3}$. The latter quantity is shown in Fig. 1 for the soft (S) and hard (H) local

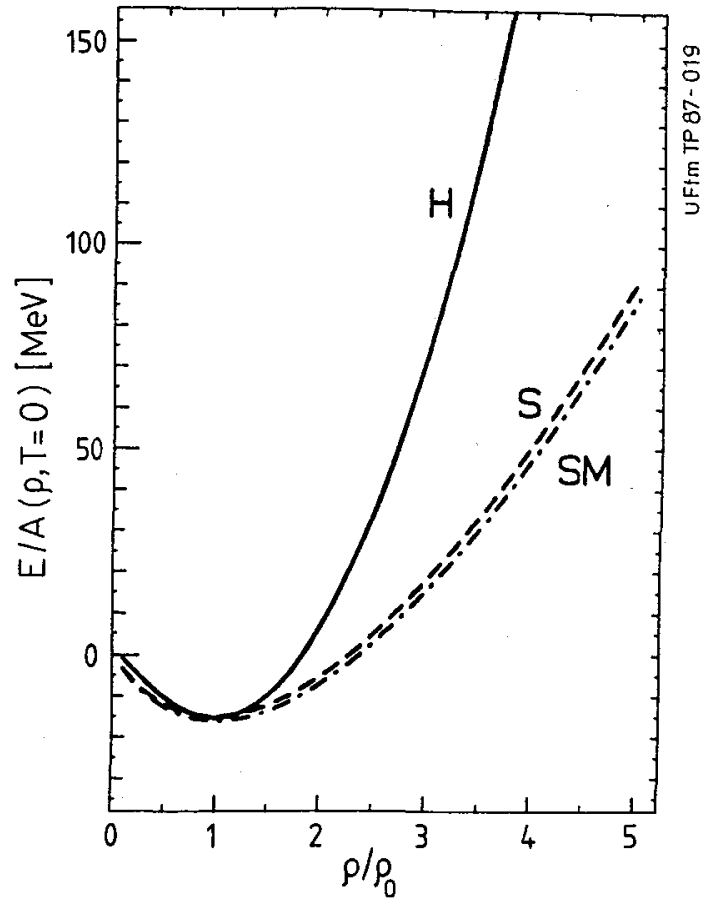

Fig. 1. The density dependence of the compression energy per particle of $\mathrm{T}=0$ infinite nuclear matter is shown for the three distinct interaction $S, S M$, and $\mathrm{H}$, respectively. Note that the momentum dependent potential SM exhibits nearly the same energies as the soft local potential S. The energy for the hard local potential $\mathrm{H}$ is much higher at the same density. 
Skyrme potentials ${ }^{3-5,11}$ and for the interaction with a momentum dependent term, which is hereafter denoted by SM. The parameters $a-\epsilon$ are given by $\alpha=-356,-390,-124: \beta=303,320,70.5 ; \gamma=1.166,1.14,2$ and $\delta=1.57, \epsilon=21.54$ for the three interactions $S, S M$ and $H$ respectively. Note that $S$ and $S M$ lead to nearly the same EOS. which is for infinite matter at $T=0$ much less repulsive than the EOS corresponding to the hard local potential H. One would natvely expect that the two different interactions $S$ and SM. which exhibit the same EOS, should yleld similar predictions for $4 \pi$ observables in high energy heavy Ion collisions. However, the main point of this paper is to demonstrate that this is not always the case: due to the large initial relative momenta of the projectile and target the momentum dependent Interaction act much more repulsively in a collision than in the $T=0$ Infinite nuclear matter case of Fig. 1.

Before discussing the influence of the momentum dependent interactions on the $4 \pi$ observables quantitatively. let us first discuss their qualitative effects for the collision dynamics. In the intial penetration phase the effect of the MDI will be most pronounced, since there the separation of the profect1le and target in momentum space is the largest. Particles are accelerated by the KDI in the transverse direction early into the reaction. This is different from the sidewards deflection occuring ith purely local interactions ${ }^{1,3}$, which occurs after stopping is achieved and the point of maximum density is reached. Hence the density pile- up in the overlap region will be lower when the MDI are present. Consequently fewer collisions w11l occur and the observables which are related to this number of collisions. namely particle production rates and longitudinal deceleration, are diminished. The transverse momentum transfer, on the other hand. will be enhanced.

Fig. 2 shows the plon, kaon and deuteron yield for the system La $(0.8 \mathrm{GeV} / \mathrm{n})+\mathrm{La}$ as a function of the impact parameter for the three 
distinct interactions discussed above. Observe that there is a $\approx 10 \%$ decrease of the pion yleld by going from the sof to the hard local potential, in agreement with earlier findings ${ }^{3}$. However, the momentum dependent interactions result in an $\approx 30 \%$ decrease of the pion yield! This strong suppression of the pion yield is due to a $30 \times$ decrease of the number of $n-n$ collisions, which in turn is due to the strong initial repulstion resulting from the large separation of the target and projectile in momentum space. These findings render the proposed determination of the EOS of Infinite nuclear matter from the pion ylelds $^{1-3}$ in heavy ion collisions alone virtually impossible.

Qualitatively similar, but quantitatively even more dramatic, results are found for subthreshold kaon production, which is of course more sensitive to the details of the reaction dynamics (Fig.2) the kaon yield drops by a factor of two when going from the sof to the hard local potential, in agreenent with the results of ref. 5 , but it drops by a factor of 4 when momentum dependent terms are introduced. 1.e. for the case SM. Again. the lower number of collisions and the deceleration, which is most pronounced for the particles with the highest momenta due to the repulsive MDI, is responsible for this behaviour. This subthreshold behaviour of the $\mathrm{K}^{+}$- production can be useful for an experimental determination of the momentum dependent part $U(\Delta p)$ of the potential.

Let us now turn to the formation of deuterons, which is calculated in impulse approximation. ${ }^{13}$ The impact parameter dependence of the deuteron-1ike to proton-like ratio $R$ is shown in Fig. 2. It exhibits nearly the same value $R=0.56$ for the two soft EOS (cases $S$ and $S M$ ), while it is about $10 x$ larger $(R=0.62)$ for the hard EOS, case $H$. Thus the deuteron formation could prove extremely useful to probe the EOS. since it is insensitive to the momentum dependent interactions for massive systems and directly reflects the EOS of Fig. 1. The entropy per participant nucleon ${ }^{14}$ is found to be similar for the different 

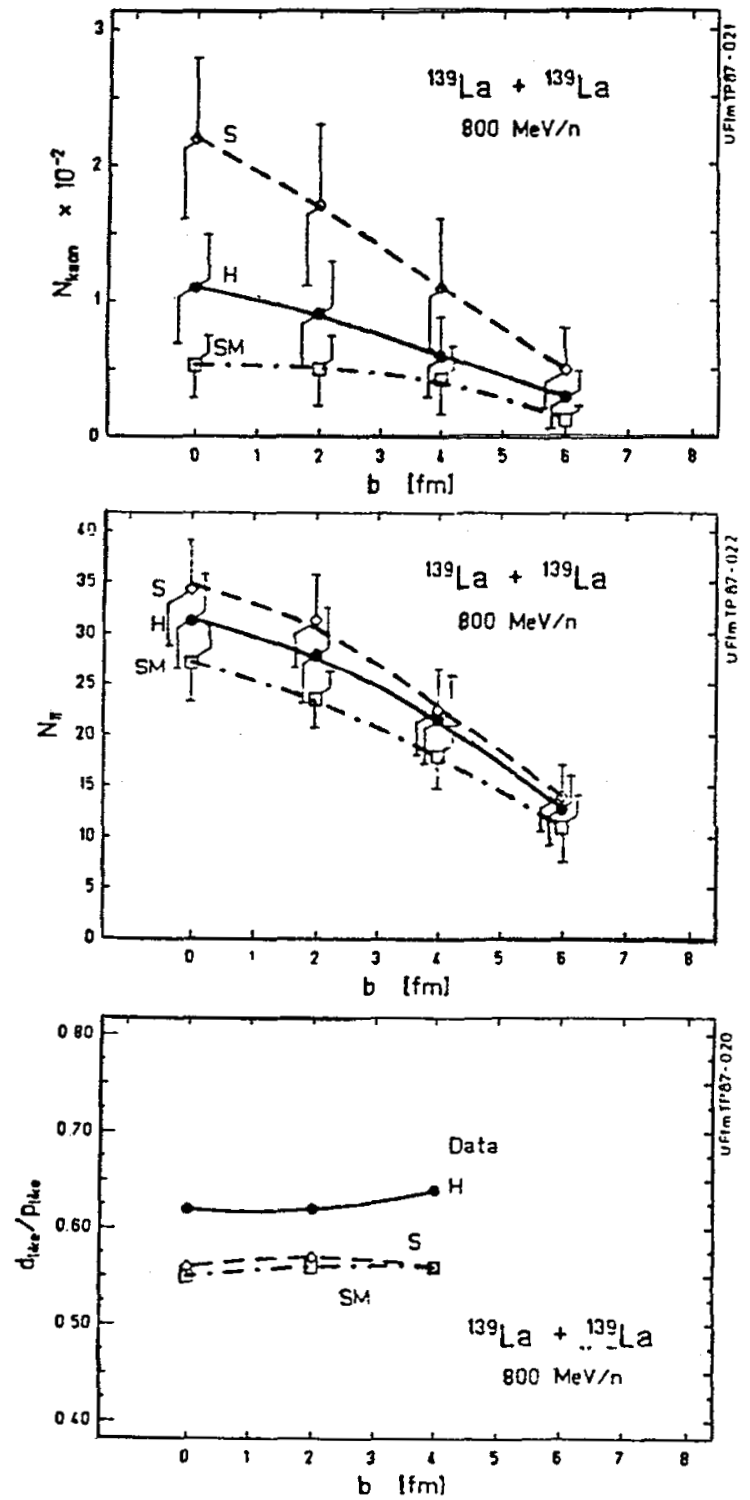

Fig. 2. The impact parameter dependence of the kaon and pion yields and the deuteron-like to proton-1ike ratio $R$ is show for the system $\mathrm{La}(0.8$ GeV/N + La using the S. SM, and $H$ interaction, respectively. Note that the MDI lead to a suppression of the pion and kaon ylelds which by far exceeds the suppression due to a hard local potential. The d/p ratios, on the other hand, are insensitive to the momentum dependent interactions. 
interactions. Recent high multiplicity selected experimental data ${ }^{14,15}$ indicate large $R$ values, $(d / p)^{\max } \approx 0.68$ for the system ${ }^{15} \mathrm{La}(0.8 \mathrm{GeV} / \mathrm{N})$ + La. This seems to indicate that the nuclear equation of state is rather hard, In accord with the results on the flow angles discussed below and with the earller findings ${ }^{1-3}$. which, however. neglected the momentum dependent terms.

Let us now turn to the collective flow observables: Fig. 3 shows the flow angles and the average transverse momentum transfer $\left\langle p_{X}\right\rangle=1 / N \Sigma$ $\operatorname{sig}(y) p_{x}(y)$ for the systems La $(0.8 \mathrm{Gev} / n)+L a$ : Note that the harder the local potential, the larger is the flow angle. This result of the QMD approach ${ }^{11}$ agrees with previous hydrodynamic and vuJ predictions 1.3 .

For momentum dependent interactions. 1.e. for the case SM, we find a slight decrease of the maximum flow angle as compared to the sof $t$ local potential $S$ alone, 1.e. here SM does not simulate a harder EOS than the soft local potentiall This can be understood as follows: The flow angle is a function of the ratio $\left\langle p_{x}\right\rangle /\left\langle p_{\|}\right\rangle$. The transverse momenta are sensitive to the $\mathrm{xDI}$. The $\left\langle\mathrm{p}_{x}\right\rangle$ values for $S X$ and $H$ are nearly the same and are about double the value obtained with the sof local potential S (Fig. 3). The reduced number of collisions for the case SM. on the other hand, results in a hlgher longitudinal momentum. which yfelds nearly the same flow angle for SW as for the soft local potential $S$. The different contributions of the local potentials and the MDI can be studied by examining the flow angle and the $p_{x}(y)$ distributions simul taneously. Hence. While no observable is at hand to date which can be related directly to the density achieved, the flow angle distributions seem to be related to the stiffness of the nuclear matter equation of state ${ }^{1.3}$. A comparison of the experimental data with the vUU model has yielded evidence for a hard EOS. ${ }^{1.3}$ Experimentally flow angles by far exceeding the present result using a sof $t$ EOS, be it with or without NDI, have been observed. ${ }^{10}$ However. for a quantitative 

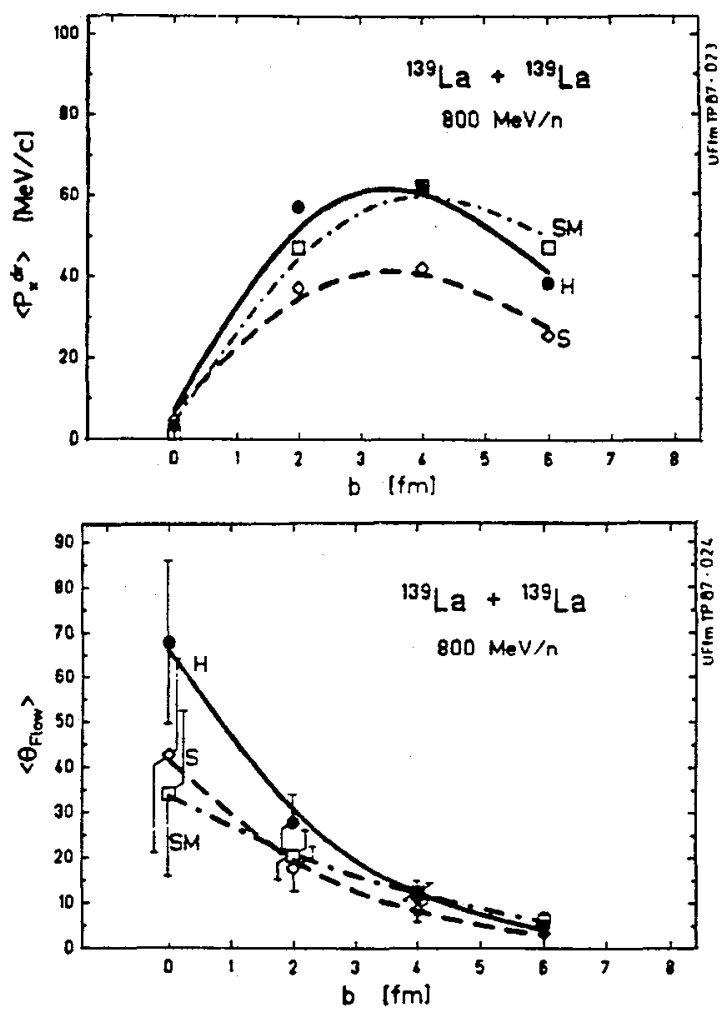

Fig. 3. The average transverse womentum $\left\langle p_{X}\right\rangle$ and the flow angle $\theta_{F}$ are shown as a function of the lumact parameter for the system La(0.8 GeV/N)+La, using the three interactions $S$. SN and H. Observe that the the $P_{X}-$ values are sensitive to the MDI. The flow angles, on the other hand, are nearly equal for the $\mathbf{S}$ and $\mathbf{S M}$ interaction, which posses the same EOS for Infinite nuclear metter at $T=0$ (See Fig. 1). Only the hard EOS seems to allow for the large flow angles observed experimentally ${ }^{10}$.

comparison with the experimental data the varlous fragments ${ }^{11}$ must be subjected to the efficlency cuts of the experimental apparatus, which might have substantial effects on the observables considered.

Me have shown that momentum dependent interactions have a large influence on the $4 \pi$ observables in heavy lon collislons. Plon and kaon yields seem to be most sensitive to the MDI and could therefore be 
useful to extract the energy and density dependence of the momentum dependent part $U(\Delta p)$ (but not the EOS per se) from experiment. From the observed large flow angles and deuteron to proton ratios we are tempted to conclude that the EOS is hard. Wuch work is needed, though. before the nuclear EOS can be pinned down conclusively from experiment. Acknowledgementg: This work was supported by the Bundesministerfum fuer Forschung und Technologie, Bonn, and the Gesellschaft fuer Schwertonenforschung (GSI), Darmstadt. J.A. acknowledges stimulating discussions with Prof. J. Huefner.

\section{References:}

[1] H.Stoecker, W.Greiner and W.Scheld, Z. Phys. A286 (1978) 121, H.Stoecker, W.Greiner. Phys. Rep. 137 (1986) 277.

[2] J.W. Harris, G. Odyniec, H. Pugh, L.S. Schroeder, M. Tinckne11, W. Rauch, R. Stock, R. Bock, R. Brockmann, A. Sandoval, H. Stroebele, R. Renfordt, D. Schall, D. Bangert, J. Sullivan. K.L. Wolf, A. Dacal, C. Guerra, M. E. Ortiz, Phys. Rev. Lett. 58 (1987) 463;

R. Stock. R. Bock. R. Brockmann, J.W. Hartis. A. Sandoval, H. Stroebele, K.E. Wolf, H.G. Pugh, L.S. Schroeder, M. Kaier, R. Renfordt, A. Dacal, M.E. Ort1z. Phys. Rev. Lett. 49 (1982) 1236.

J. Harris et al. Phys. Lett. 153B (1985) 377.

R.Stock, Phys. Rep. 135 (1986) 259.

[3] H. Kruse, B.V. Jacak and H.Stoecker. Phys.Rev.Lett. 54 (1985) 289. Their results disagree with those of G. F. Bertsch, H. Kruse and S. DasGupta, Phys. Rev. C29 (1984) 673. who had claimed that there is no sensitivity of the pion yield on the EOS.

[4] J.J. Molitoris and H. Stoecker. Phys.Rev.C32 (1985) 346. Phys. Lett. 162B (1985) 47. Prog. Part. Nucl. Phys. 15(1385) 239, and Phys. Rev. C. In print. 
[5] J.Aichelin and C.M. Ko. Phys. Rev. Lett. 55 (1985) 2661.

For a comparison between various methods see e.g.

J.Aichelin and H.Stoecker. Phys. Lett. B163 (1985) 59;

J.Aichelin, Phys. Rev. C33 (1986) 537

[6] T.L. Alnsworth, E. Baron, G. E. Brown, J. Cooperstein, M. Prakash, Nuc1. Phys. A, in print.

C. Gale. G. Bertsch. S. DasGupta have reported orally at the workshop on the nuclear squation of state. Berkeley, CA. Kay 1986. that they find no effect of the $x D I$ on the $p_{x}$ - distribution.

This is in contrast to the conclusions presented here.

[7] A.R. Bodmer, C. Panos, A.D. Mackellar, Phys.Rev.C22 (1980) 1025:

L. Filets. Y. Yariv and R. Chestnut, Nucl. Phys. A301 (1978) 359;

[8] R. Y. Gusson, P.G. Reinhardt, J.J. Molftoris, H. Stoecker, M. Strayer. W. Greiner, Phys. Rev. Lett. 55 (1985) 2786.

[9] J.J. Molitoris, J.B. Hoffer, H. Kruse and H. Stoecker. Phys. Rev. Lett, 53 (1984) 899.

[10] H.G. RItter et al., Nucl. Phys. 3C (1985) 1447;

H.A. Gustafsson, H.H. Gutbrod, B. Kolb, H. Loehner, B. Ludewigt, A.M. Poskanzer, T. Renner, H. Riedesel, H.G. Ritter, A. Warwick and H. Wiemenn, Phys.Lett. B142 (1984) 141.

P. Danielewicz, G. Odyntec, Phys. Lett. 1578 (1985) 146.

[11] J. Alchelin and H. Stoecker. Phys. Letr. B176 (1986) 14

A. Rosenhauer, J. Alchelin, H. Stoecker, M. Greiner. J. de Physique C4 (1986) 395, and to be published.

[12] J.P. Jeuhenne, A. Lejeune, C. Kahaux, Phys. Rep. 25 (1876) 85

[13] J. Alchelin, E. Remler, Phys. Rev. C, in press, and refs, therein.

[14] K.G.R. Doss et al, Phys. Rev. C32 (1985) 116

D. Hahn. H. St jecker. LBL preprint 22378 (1986).

[15] H. Stroebele, CSI- Report 86-10, and to be published, S. Hayasht et al. Columbia University preprint, and private communications . 